\title{
Promoting and improving three Rs practice: the Korean guidelines
}

\author{
Byung In Choe ${ }^{1, *} \mathcal{E}$ Gwi Hyang Lee ${ }^{1,2}$ \\ ${ }^{1}$ Division of Institutional Review and Research Ethics, Nicholas Cardinal Cheong Graduate School for Life, The Catholic University of \\ Korea, Seoul 06591, ${ }^{2}$ BIC Study, Seoul 06647, Korea
}

Scientists planning research that involves the use of animals are required to examine the possibilities for replacement, reduction, or refinement (the Three Rs), and their protocol must be reviewed by the Institutional Animal Care and Use Committee. Good scientific practice involving appropriate search techniques on the available Three Rs resources is essential for both ethical and scientific reasons. Appropriate experimental design and statistical analysis techniques are particularly necessary for research involving the use of animals, because this can improve animal welfare and scientific outcomes, as well as saving animal lives. There are a number of resources to help researchers improve their search techniques, experimental design strategies, and their reporting of research involving the use of animals. However, there is little specific information or resources on the Three Rs alternatives that is readily available in the Korean language. This paper outlines the common errors made by submitting researchers that have been repeatedly observed during the ethical review of experimental protocols over the last ten years, and provides information on the Korean resources available to promote good scientific practice. This could help to bridge the gap between Korean scientists and animal welfare advocates assisting scientists to improve ethical considerations and conduct responsible research. [BMB Reports 2017; 50(12): 610-614]

\section{INTRODUCTION}

Any research proposal involving the use of animals in Korea requires regulatory approval. This year marks the 10th anniversary of the proclamation of the Animal Protection Act in Korea for the humane use of animals in science, i.e.

*Corresponding author. Tel: +82-2-2258-7395; Fax: +82-70-40997884; E-mail: bichoe@catholic.ac.kr

https://doi.org/10.5483/BMBRep.2017.50.12.225

Received 30 October 2017

Keywords: Education, Experimental design, Korean guidelines, Reporting of animal research, Search techniques, Three Rs applying the Three Rs principles of Russell and Burch, and having an ethical review process prior to conducting animal experiments in research, testing, and education (1). Since 2008, scientists planning research involving the use of animals have been required to examine the possibilities for replacement, reduction, or refinement (the Three Rs) according to two Korean laws - the Animal Protection Act (2008) and the Laboratory Animal Act (2009) (2). Through this process, researchers can prevent unintended and unnecessary duplication of animal experimentation, and may acquire improved methods with regard to experimental design that help to reduce or replace animal use, and refine the procedures to minimize pain and distress to animals $(2,3)$. The researchers must be properly trained, and the protocol must be reviewed by the Institutional Animal Care and Use Committee (hereafter, the IACUC), prior to starting animal use $(2,4,5)$. It is important that experiments are well designed and correctly analysed, in order to minimise pain inflicted, and maximize the chance of obtaining scientifically valid results (3). Appropriate experimental design and statistical analysis techniques are key means of minimising the use of animals in research $(3,6)$. Scientific papers should report sufficient relevant information about the experimental objectives, animal characteristics, experimental methods used, and the results obtained, in order to critically assess the findings and both the scientific and ethical implications of a study, or to allow the work to be repeated (6).

There is little specific information and few resources on the Three Rs available in Korea (2). Good scientific practice with appropriate experimental design and statistical analysis techniques are key means of minimizing the use of animals in research. This is particularly necessary for scientific research involving the use of animals, as poorly designed experiments and reporting omissions can raise both ethical and scientific concerns (7).

In 2016, there were 364 IACUCs registered with the relevant Korean government agency - which is the Animal Protection \& Welfare Division of the Animal and Plant Quarantine Agency (1). The authors have been acting IACUC members and in more than five different types of institutions (academic, medical, government and public agencies, education and training, and research institutions) since 2008, but the quality of animal 
protocols has seen little improvement over this time. A range of errors are commonly made by researchers submitting the protocols, and these have been repeatedly seen by the authors, during the protocol review processes in which they have been involved over the past ten years. They are: 1) misinterpreting the concept of the Three Rs; 2) possessing limited search skills for the Three Rs methods; 3) having a lack of awareness of the most appropriate Three Rs-specific information resources; 4) neglecting to search for the most up-to-date Three Rs information available; 5) displaying a poor selection of relevant search terms and keywords, and 6) presenting poor experimental design. In this context, the authors will review these findings into three key categories on the problems associated with the interpretation of the Three Rs concept, search techniques, and experimental design.

The authors have prepared a number of Korean versions of international guidelines on this topic, in order to help with the language barrier and the acquisition of basic information on the Three Rs, to provide a high-quality, credible source of information and resources, and to promote Three Rs practice in Korea. They are Korean versions of: 1) The EURL ECVAM Search Guide: Good Search Practice on Animal Alternatives; 2) an abridged version of The Three Rs and Humanity Criterion, written by Professor Michael Balls in 2009, and published by FRAME; and 3) the ARRIVE (Animal Research: Reporting of In Vivo Experiments) Guidelines.

\section{MISINTERPRETING THE CONCEPT OF THE THREE Rs: THE THREE Rs AND THE HUMANITY CRITERION}

The term "alternatives" is often misinterpreted as only "replacement" in Korean, and considered solely as "direct or absolute replacement". Reduction in the numbers of animals used through maximizing the quality of the experimental design is often misinterpreted as meaning the use of the fewest animals possible. However, poor experiments that use too few animals may fail to pick up biologically important effects, while those researchers who use them incorrectly or wastefully may get invalid results, while subjecting the animals to unnecessary pain, distress, or lasting harm $(6,7)$. The Three Rs concept comes from the book The Principles of Humane Experimental Technique, written by W.M.S. Russell and R.L. Burch in 1959 (8). An abridged version, The Three Rs and Humanity Criterion, was written by Professor Michael Balls in 2009, and published by FRAME (9). The authors published a Korean version of the abridged book in 2016.

\section{LIMITED SEARCHING SKILLS FOR THE THREE Rs METHODS: GOOD SEARCH PRACTICE ON ANIMAL ATERNATIVES}

Searching for Three Rs alternatives is not a structural part of the research process, and search skills are often limited. Common errors made by researchers submitting the protocols are 1) possessing limited search skills for the Three Rs methods; 2) having a lack of awareness of the most appropriate Three Rs-specific information resources; 3) neglecting a search for the most up-to-date Three Rs information available; and 4) displaying a poor selection of relevant search terms and keywords.

The findings of the surveys on the current practice of searching for information on the Three Rs methods conducted among scientists of the Radboud University Medical Center in 2009 (6) and the Netherlands national survey in 2010 showed a gap between the statutory requirement to apply Three Rs methods, and the lack of criteria to search for them (10).

Based on our national survey of Korean IACUC members and researchers in 2012 and 2016, assessing the practice of literature searching to comply with the need to use Three Rs alternatives, under $25 \%$ of the respondents had no relevant experience of searching for literature on Three Rs alternative methods. Over $80 \%$ of the respondents stated that specific training on Three Rs search skills should be included in the required training (paper in progress). Despite the tremendous amount of information on the Three Rs that is available on-line, it can be very difficult to obtain the specific information desired to replace, reduce, and refine animals used for teaching and research, as required by legislation in Korea and elsewhere $(2,5)$.

The Joint Research Centre's European Union Reference Laboratory for Alternatives to Animal Testing (EURL ECVAM) published the EURL ECVAM Search Guide: Good Search Practice on Animal Alternatives in 2012, and re-published an entirely updated second version in 2013. This guideline provides search procedures and user guidance to facilitate the location of the desired information on the Three Rs alternatives (11). It supports untrained database users in finding high quality information on relevant strategies and methods that can be used as alternatives to animal experiments, from the wide range of sources available on the web, for example, where comprehensive searches for alternatives are required as part of authorisation processes for animal experiments, and where regulatory requirements mandate the application of the Three Rs (12).

The authors developed a Korean guidance on the Three Rs search by modifying the EURL ECVAM Search Guide, publishing the Effective Search Strategies to comply with the 3Rs: 7 Golden Steps in 2014, and re-publishing the second updated version in 2016. In addition, the supplement guidebook was published, which applied Korean laws and research culture.

\section{THE VALUE OF SCIENTIFIC STUDY DESIGN AND REPORTING: THE ARRIVE GUIDELINES}

Based on a survey of animal research articles featuring experiments using mice, rats, and non-human primates in the U.S. and U.K., Kilkenny et al. reported that a minimum 
amount of relevant information must be included in scientific publications to ensure that the methods and results of a study can be reviewed, analyzed, and repeated (13). The survey identified the importance of scientific publications, and the shared responsibility of authors, peer reviewers, and journal editors to ensure that published studies describe their methods and results comprehensively, accurately, and transparently $(10,13)$

The ARRIVE Guidelines were developed in 2003 by the National Centre for the Replacement, Refinement and Reduction of Animals in Research (NC3Rs), in collaboration with researchers, statisticians, and journal editors. The objective of the ARRIVE Guidelines is to maximize the amount of high-quality data obtained from the minimum number of experiments, to avoid unnecessary animal use, and to ensure comprehensive and transparent reporting. The guidelines consist of a 20-item-checklist of the key information that should be included in a manuscript (Title, Abstract, Introduction, Methods, Results, and Discussion), to ensure comprehensive and transparent reporting in two A4 pages. It is widely accepted that applying the Three Rs to experiments involving the use of animals is consonant with good scientific practice, and currently over 300 journals advocate high reporting standards and the most efficient way to improve the reporting of a manuscript (13-16).

The authors prepared the Korean version of the ARRIVE Guidelines to raise awareness of the issues among scientists, animal welfare advocates, ethical review committees, institutions, and government agencies, and to explain the value of good scientific study design. The Korean ARRIVE Guidelines were in preparation for almost two years after the first translation submission. It proved to be quite a long process, and a diverse range of experts were involved in checking the draft translation (English original to Korean) and back translation (Korean translation to English). The purpose of this detailed process is to determine which key points to consider when translating English to another language, and vice versa. This could also help raise awareness within the Korean scientific community, and explains why the guidelines are important, and where they come from. The Korean ARRIVE Guidelines are now available on the NC3Rs website (https:// www.nc3rs.org.uk/arrive-guidelines\#translations) and the BIC Study (http://www.bicstudy.org/main/main.php). The ARRIVE Guidelines are now available in 8 different languages.

There are also the PREPARE (Planning Research and Experimental Procedures on Animals: Recommendations for Excellence) Guidelines for planning animal experiments, which are complementary to the ARRIVE Guidelines and other guidance for reporting experiments (17). The PREPARE Guidelines were developed at the Norwegian School of Veterinary Science, and are designed to be applicable to all types of animal research and testing, including field studies. They also contain topics concerning the management of animal facilities, since in-house experiments are dependent upon their quality and standards (17). The guidelines cover 15 main topics:

1. Literature searches

2. Legal issues

3. Ethical issues, harm-benefit assessment and humane endpoints

4. Experimental design and statistical analysis

5. Objectives and timescale, funding and division of labour

6. Facility evaluation

7. Education and training

8. Health risks, waste disposal and decontamination

9. Test substances and procedures

10. Experimental animals

11. Quarantine and health monitoring

12. Housing and husbandry

13. Experimental procedures

14. Humane killing, release, reuse or rehoming

15. Necropsy $(18,19)$.

The authors are currently reviewing the Korean version of the PREPARE Guidelines, produced by Norecopa, Norway's National Consensus Platform, founded October 10, 2007.

\section{EDUCATION AND TRAINING FRAMEWORK}

Education and training is very important for researchers and IACUC members to successfully carry out their role in practice. Both Korean regulatory agencies, i.e. the Animal and Plant Quarantine Agency, and the Korean Association for Laboratory Animals, provide regular training based on the legal requirements $(20,21)$. The practical topics and educational sessions should prove beneficial in overcoming the current challenges in the field, and provide regulatory updates for the more experienced groups $(2,4)$. However, search technique for the Three Rs methods is not a mandatory part of the training curriculum for compliance with the requirements of the Korean laws and other regulations - e.g. the Animal Protection Act and Laboratory Animal Act $(22,23)$.

The IACUC appointment framework is for two-year terms, and since 2012 requires a four-hour course certificate issued by the Animal and Plant Quarantine Agency (16). Researchers planning to use animals for scientific purposes must complete a six-hour qualification course. Both regulatory agencies mostly offer 'instructor-led training' and off-line training. Educational opportunities should be provided, when they are needed (16). The authors addressed how different types of training method that take into consideration an individual's schedule and their interest in the topics in the paper published in 2014, and a web-based teaching method has now been approved as a type of qualification training course, according to the Enforcement Rule of the Animal Protection Act (Chapter, Article 26 (Qualification of IACUC Members) Item 4 (22), and the Standard of Operation of the Animal Protection \& Welfare Curricula or Animal Experiment Training Course, effective as of August 18, 2017 (Animal and Plant Quarantine Agency 
Notice 2017-33) (20). The BIC Study is now accredited by the Animal and Plant Quarantine Agency as the online education provider, effective as of September 29, 2017 (24). To support the acquisition of basic information on the Three Rs, the authors have, since 2013, been developing an independent, non-profit platform website to facilitate the sharing of available educational resources. Educating scientists and IACUC members and helping them embrace the use of alternatives has proved a good place to start, and is key to giving them the skills to find useful information.

\section{LIMITATIONS AND CHALLENGES}

The Korean Guidelines were translated by the authors, and the quality of Korean translation was subsequently reviewed in draft form by individuals chosen for their diverse perspectives, including some within the relevant government agencies. The purpose of this diverse independent review is to provide critical comments, and to ensure that the translation meets the national standard for objectivity, evidence, and responsiveness, and that it also meets the international standard. Korean is an agglutinative language in its morphology of Subject-Object-Verb syntax. The most important thing in translation is to convey the correct message (if not, the nearest possible message). One word can have different meanings in different research cultures and societies, and this is especially true in academic society. Therefore, it is necessary to interpret the foreign language word in its relevant context, instead of translating it directly, and often a more specialized Korean word is then needed for the translation, rather than Korean in general. The Korean Guidelines are focused on a scientific and academic group of readers. Therefore, providing contextual meaning of the original message is more important than direct linguistic translation. Any disagreements or differences in interpretation of the guidelines may subsequently improve the translation by further positive discussion.

\section{CONCLUSIONS AND THE WAY FORWARD}

Overall, Korea is moving forward in applying the Three Rs principles and improving laboratory animal welfare. The promotion and protection of research animals is one of the core competencies of well-educated involved personnel. Training in proper search techniques, and having user-friendly systems on the practical application of the Three Rs, are essential when considering the use of animals in research and teaching.

After dedicating our efforts to the long process of producing the Korean Guidelines, our hope now is to ensure their effective use in qualification courses for researchers and IACUC members, and that they apply the guidelines in their research practice. The Korean Guidelines could help researchers think about the issues early on in the scientific process, well before the writing-up stage, and translate findings from previous animal experiments, thus avoiding unnecessary animal use. The IACUC members may also refer to the Guidelines as a checklist, to ensure that a submitted protocol meets the requirements.

The authors believe that good science is based on good ethical practice. Good scientific practice, utilizing all available resources, with appropriate searching for Three Rs alternative methods, and good experimental design and statistical analysis techniques, is particularly necessary for research involving the use of animals. It is also essential for ethical and scientific reasons, because this can improve animal welfare and scientific outcomes, as well as saving animal lives. It is time to take education on the Three Rs to the next level, by considering "Why?", as a follow-on from "How?". It is our hope that these Korean Guidelines will gain ever increasing attention from researchers and IACUC members.

\section{ACKNOWLEDGEMENTS}

The authors would particularly like to acknowledge the contribution of the Korean translation review. They are: Dr. Byeong Han Lee, Laboratory Animal Center of the Osong Medical Innovation Foundation; Dr. Yun-Kyung Moon and Mr. Moon Seok Yoon of the Animal Protection \& Welfare Division, Animal and Plant Quarantine Agency; Prof. Jae Hak Park of Seoul National University; and Prof. Jin Soo Han of KonKuk University of Korea.

\section{CONFLICTS OF INTEREST}

The authors have no conflicting interests.

\section{REFERENCES}

1. Choe BI and Lee GH (2017) Progress and Challenges of the 3Rs Resource Platform in Korea: The way forward. WC10 Poster presentation, Seattle, USA

2. Choe BI and Lee GH (2013) Searching and review on the Three Rs information in Korea: time for quality assessment and continued education. BMB Rep 46, 335-337

3. Kilkenny C, Parsons N, Kadyszewski E et al (2009) Survey of the Quality of Experimental Design, Statistical Analysis and Reporting of Research Using Animals. PLoS One 4, e7824

4. Anderson LC (2007) Institutional and IACUC Responsibilities for Animal Care and Use Education and Training Programs. ILAR J 48, 90-95

5. Nesdill D and Adams KM (2011) Literature search strategies to comply with institutional animal care and use committee review requirements. J Vet Med Educ 38, 150-156

6. Leenaars M, Savenije B, Nagtegaal A, van der Vaart L, Ritskes-Hoitinga M (2009) Assessing the search for and implementation of the Three Rs: a survey among scientists. Altern Lab Anim 37, 297-303

7. Leenaars M, Hooijmams CR, van Veggel $\mathrm{N}$ et al (2012) A 
step-by-step guide to systematically identified all relevant animal studies. Lab Anim 46, 24-31

8. Russell WMS and Burch RL (1959) The Principles of Humane Experimental Technique. London: Metheun; Available: http://altweb.jhsph.edu/pubs/books/humane_exp/ het-toc\#. (Accessed 20.10. 2017)

9. Choe $\mathrm{BI}$ and Lee GH (2016) Korean Translation of the Three Rs and the Humanity Criterion. Altern Lab Anim 44, 515-517

10. van Luijk J, Cuijpers $Y$, van $\operatorname{der}$ Vaart $L$, Leenaars $M$, Ritskes-Hoitinga M (2011) Assessing the search for information on Three Rs methods, and their subsequent implementation: a national survey among scientists in the Netherlands. Altern Lab Anim 39, 429-447

11. Joint Research Center, European Union Reference Laboratory for alternatives to animal testing (EURL ECVAM). https://ec.europa.eu/jrc/en/publication/books/eurl-ecvam-s earch-guide-good-search-practice-animal-alternatives (Accessed 06.11.17)

12. Hudson-Shore M (2012) Searching Effectively for Three Rs information. Altern Lab Anim 40, 22-23

13. Kilkenny C, Browne WJ, Cuthill IC, Emerson M, Altman DG (2010) Improving bioscience research reporting: The ARRIVE Guidelines for Reporting Animal Research. PLoS Biol 8, e1000412

14. NC3Rs. https://www.nc3rs.org.uk/arrive-guidelines (Accessed 06.11.17)

15. The ARRIVE guidelines: speaker notes, http://www.nc3rs.
org.uk/sites/default/files/documents/Guidelines/ARRIVE\%2 0Guidelines\%20Speaker\%20Notes.pdf (Accessed 26. 07. 2017)

16. Choe $\mathrm{BI}$ and Lee $\mathrm{GH}$ (2014) Individual and collective responsibility to enhance regulatory compliance of the Three Rs. BMB Rep 47, 179-183

17. Hooijmans CR, Leenaars M and Ritskes-Hoitinga M (2010) A gold standard publication checklist to improve the quality of animal studies, to fully integrate the Three Rs, and to make systematic reviews more feasible. Altern Lab Anim 38, 167-182

18. Smith AJ, Clutton RE, Lilley E, Hansen KEA, Brattelid T (2017) PREPARE: guidelines for planning animal research and testing. Lab Anim [Epub ahead of print]

19. Norecopa. https://norecopa.no/PREPARE. (Accessed 26. 07.2017)

20. Animal and Plant Quarantine Agency. http://www.animal. go.kr/aec/main/main.jsp. (Accessed 26. 07.2017)

21. Korean Association for Laboratory Animals. https://www. kafla.kr:466/(Accessed 26. 07.2017)

22. Animal Protection Act. http://www.law.go.kr/eng/engLsSc.do? menuld $=1$ \&query $=\%$ E3 $\% 85 \%$ A3LABORATORY + ANIM $\mathrm{AL}+\mathrm{ACT} \& \mathrm{x}=29 \& \mathrm{y}=23 \#$ liBgcolor10 (Accessed 06.11.17)

23. Laboratory Animal Act. http://www.law.go.kr/eng/engLsSc.do? menuld $=1$ \&query $=\%$ E3 $\% 85 \%$ A3LABORATORY + ANIM $\mathrm{AL}+\mathrm{ACT} \& \mathrm{x}=29 \& \mathrm{y}=23 \#$ liBgcolor0 (Accessed 06.11.17)

24. BIC Study. https://www.bicstudy.org (Accessed 06.11.15) 